PROCEEDINGS OF THE

AMERICAN MATHEMATICAL SOCIETY

Volume 138, Number 11, November 2010, Pages 4149-4155

S 0002-9939(2010)10424-0

Article electronically published on May 26, 2010

\title{
CONTINUOUS LINEAR EXTENSION OF FUNCTIONS
}

\author{
A. KOYAMA, I. STASYUK, E. D. TYMCHATYN, AND A. ZAGORODNYUK
}

(Communicated by Nigel J. Kalton)

\begin{abstract}
Let $(X, d)$ be a complete metric space. We prove that there is a continuous, linear, regular extension operator from the space $C_{b}^{*}$ of all partial, continuous, real-valued, bounded functions with closed, bounded domains in $X$ to the space $C^{*}(X)$ of all continuous, bounded, real-valued functions on $X$ with the topology of uniform convergence on compact sets. This is a variant of a result of Kunzi and Shapiro for continuous functions with compact, variable domains.
\end{abstract}

\section{INTRODUCTION}

There is a long history of improvements to the Tietze-Urysohn extension theorem. Dugundji [4 proved that if $A$ is a closed subset of a metric space $X$, then there is a continuous, linear, regular extension operator from $C(A)$, the space of continuous real-valued functions on $A$ with the topology of pointwise convergence, to the space $C(X)$. His operator is also continuous with respect to the topology of uniform convergence on $C(A)$ and $C(X)$.

Kuratowski [7] first considered the space of all continuous partial functions whose domains are closed subsets of a metric space $X$. The question of existence of operators extending partial functions with variable domains then arose naturally. A non-linear extension operator for partial functions with compact domains was constructed by Stepanova [12.

Kunzi and Shapiro [6 improved the theorems of Dugundji and Stepanova to encompass functions with compact variable domains as follows:

Let $(X, d)$ be a metric space and let

$$
C_{v c}=\{f: A \rightarrow \mathbb{R} \mid A \subset X \text { is compact and } f \text { is continuous }\} .
$$

Then $C_{v c}$ is a metric space where the distance between two functions $f$ and $g$ is given by the Hausdorff distance between their graphs, which are closed, bounded subsets of $X \times \mathbb{R}$. Let $C^{*}(X)$ denote the set of continuous, bounded, real-valued functions on $X$.

Received by the editors September 10, 2009 and, in revised form, November 20, 2009 and February 3, 2010.

2010 Mathematics Subject Classification. Primary 54C20, 54C30; Secondary 54E40.

Key words and phrases. Extension of functions, continuous linear operator, metric space.

The second, third, and fourth authors were supported in part by NSERC grant No. OGP 0005616. 
Theorem $1.1([6])$. Suppose that $X$ is a metrizable space and that the set $C^{*}(X)$ is endowed with the topology of uniform convergence. Then there exists a continuous operator $\Phi: C_{v c} \rightarrow C^{*}(X)$ with the following properties:

1) $\left.\Phi(f)\right|_{\operatorname{dom} f}=f$ for every $f \in C_{v c}$;

2) $\Phi$ is regular, i.e.

$$
\|\Phi(f)\|=\|f\|=\max \{|f(x)| \mid x \in \operatorname{dom} f\}
$$

for every $f \in C_{v c}$ and $\Phi\left(\mathbf{1}_{A}\right)=\mathbf{1}_{X}$ for every compact subset $A$ of $X$;

3) for every compact $A \subset X$ the restriction $\left.\Phi\right|_{C(A)}$ is a linear operator, i.e. $\Phi(\alpha f+\beta g)=\alpha \Phi(f)+\beta \Phi(g)$ for $\alpha, \beta \in \mathbb{R}$ and $f, g \in C(A)$.

It has not been proved that the Kunzi-Shapiro operator preserves uniformly continuous functions if $X$ is not compact.

Let

$$
\begin{array}{r}
C_{u b}^{*}=\{f: A \rightarrow \mathbb{R} \mid f \text { is uniformly continuous and bounded, } \\
A \subset X \text { is closed and bounded }\} .
\end{array}
$$

As in the case of $C_{v c}$ define the distance between two functions in $C_{u b}^{*}$ to be the Hausdorff distance between their graphs. Denote by $C_{u}^{*}(X)$ the family of uniformly continuous, bounded, real-valued functions on $X$. For a bounded space $X$ the following result is known:

Theorem $1.2([3])$. Let $X$ be a bounded metric space. There exists a regular, positive homogeneous extension operator $u: C_{u b}^{*} \rightarrow C_{u}^{*}(X)$ which is continuous with respect to the topology of uniform convergence on $C_{u}^{*}(X)$.

Note that extension operator constructed in 3 is not additive and therefore is not linear. The Kunzi-Shapiro theorem's proof seems to depend essentially on the compactness of domains of partial functions. Of course all of the partial functions considered by Kunzi and Shapiro are uniformly continuous.

It is known that Hausdorff metric convergence of graphs of continuous functions with common domain implies pointwise convergence as well as uniform convergence on compact sets but does not imply the uniform convergence of these functions. However, if the limit function is uniformly continuous, then this last implication is true (see [2, 9]). In the current paper we prove a variant of the result of Kunzi and Shapiro for the case of bounded continuous functions defined on all closed, bounded subsets of a complete metric space. We construct an extension operator which is linear, regular and continuous with respect to the topology of uniform convergence on compact sets on $C^{*}(X)$. In short, we weaken the compactness condition on partial domains in the Kunzi-Shapiro result, but the trade-off is that our operator is continuous with respect to the topology of uniform convergence on compact sets on $C^{*}(X)$ rather than the topology of uniform convergence.

Note that there is a vast literature devoted to extensions of functions with special properties (for instance see [13] for a counterpart of the Kunzi-Shapiro theorem for pseudometrics). 


\section{Preliminaries}

Let $(X, d)$ be a metric space and $\exp _{b}(X)$ the space of non-empty, closed and bounded subsets of $X$ with Hausdorff metric $H$. Let $\rho$ be the metric on the product space $X \times \exp _{b}(X)$ given by the formula

$$
\rho[(x, A),(y, B)]=d(x, y)+H(A, B)
$$

for every $x, y \in X$ and $A, B \in \exp _{b}(X)$. Let $\pi_{1}$ and $\pi_{2}$ stand for the projection maps in the product space $X \times \exp _{b}(X)$.

For $A \in \exp _{b}(X)$ let $C^{*}(A)$ denote the family of continuous, bounded, real-valued functions on $A$. Let

$$
C_{b}^{*}=\bigcup\left\{C^{*}(A) \mid A \in \exp _{b}(X)\right\} .
$$

We identify each $f \in C_{b}^{*}$ with its graph

$$
\Gamma_{f}=\{(x, f(x)) \mid x \in \operatorname{dom} f\},
$$

which is a bounded and closed subset of $X \times \mathbb{R}$ (here $\operatorname{dom} f$ denotes the domain of $f)$. Let $\tilde{d}$ be the metric on $X \times \mathbb{R}$ defined by

$$
\tilde{d}\left((x, t),\left(x^{\prime}, t^{\prime}\right)\right)=d\left(x, x^{\prime}\right)+\left|t-t^{\prime}\right|
$$

for $(x, t),\left(x^{\prime}, t^{\prime}\right) \in X \times \mathbb{R}$. Let $\tilde{H}$ be the Hausdorff metric on $\exp _{b}(X \times \mathbb{R})$ induced by $\tilde{d}$. For $f, g \in C_{b}^{*}$ let the distance from $f$ to $g$ be given by $\tilde{H}\left(\Gamma_{f}, \Gamma_{g}\right)$.

We say that $e: C_{b}^{*} \rightarrow C^{*}(X)$ is an extension operator if for every $f \in C_{b}^{*}$ we have $\left.e(f)\right|_{\operatorname{dom} f}=f$.

We say that $e$ is regular if

$$
\|e(f)\|=\sup \{|e(f)(x)| \mid x \in X\}=\sup \{|f(x)| \mid x \in \operatorname{dom} f\}=\|f\|
$$

for each $f \in C_{b}^{*}$ and $e\left(\mathbf{1}_{A}\right)=e\left(\mathbf{1}_{X}\right)$ for every $A \in \exp _{b}(X)$ where $\mathbf{1}_{A}$ is the constant map on $A$ with value equal to 1 .

Finally, $e$ is linear if $e(a f+b g)=a e(f)+b e(g)$ for all $f, g \in C_{b}^{*}$ with $\operatorname{dom} f=$ $\operatorname{dom} g$ and $a, b \in \mathbb{R}$.

For a topological space $Y$ denote by $\mathcal{P}(Y)$ the space of all regular, positive, Borel, probability measures on $Y$ endowed with the weak topology with respect to $C^{*}(Y)$, the Banach space of all continuous, bounded, real-valued functions with the sup-norm topology. For a topological space $Z$ and a map $m: Y \rightarrow Z$ a family $\left\{\mu_{z}\right\}_{z \in Z}$ of measures from $\mathcal{P}(Y)$ is called a fiberwise measure on $m$ if

(i) $\mu_{z}$ depends continuously on $z \in Z$ (this means that $\int_{Y} \psi d \mu_{z}$ is a continuous function on $Z$ for every $\psi \in C^{*}(Y)$ );

(ii) $\operatorname{supp} \mu_{z}=\bigcap\left\{C \subset Y \mid C\right.$ is closed and $\left.\mu_{z}(C)=\mu_{z}(Y)\right\}$ is a subset of $m^{-1}(z)$ for every $z \in Z$.

A map $m$ that admits a probability fiberwise measure is called a Milyutin map. A fiberwise measure $\left\{\mu_{z}\right\}_{z \in Z}$ on $m$ is called atomless if $\mu_{z}(y)=0$ for every $z \in Z$ and $y \in Y$. We are going to use the following result due to Ageev and Tymchatyn:

Theorem 2.1 ([1]). For each metric space $Z$ there exists a perfect (i.e., closed with compact fibers), atomless, Milyutin map $m: Y \rightarrow Z$ of a zero-dimensional metric space $Y$ (i.e., the covering dimension $\operatorname{dim} Y$ is 0 ). 


\section{The MAIN Result}

The following theorem is the main result of this paper.

Theorem 3.1. Let $(X, d)$ be a complete metric space. There exists a regular, linear extension operator $e: C_{b}^{*} \rightarrow C^{*}(X)$. This operator is continuous with respect to the topology of uniform convergence on compact sets on $C^{*}(X)$.

Proof. We will need to define a certain multi-valued map in order to construct the extension operator $e$.

Let $K=\bigcup\left\{A \times\{A\} \mid A \in \exp _{b}(X)\right\}$ with metric $\rho$. Then $K$ is closed in $X \times \exp _{b}(X)$.

Let $\mathcal{U}$ be an open, locally finite cover of $\left(X \times \exp _{b}(X)\right) \backslash K$ such that $U \in \mathcal{U}$ implies $\operatorname{diam}(U)<\frac{1}{2} \rho(z, U)$ for each $z \in K$.

By Theorem 2.1let $m: Y \rightarrow X \times \exp _{b}(X)$ be a Milyutin map with compact fibers of a 0 -dimensional metric space $Y$ with a continuous family of fiberwise probability measures on $Y$,

$$
\left\{\mu_{(x, A)}\right\}_{(x, A) \in X \times \exp _{b}(X)},
$$

where the support of $\mu_{(x, A)}$ is contained in $m^{-1}(x, A)$ for each $(x, A) \in X \times \exp _{b}(X)$.

For $U \in \mathcal{U}$ let

$$
\begin{array}{r}
W_{U}=\{(a, A) \in K \mid \text { there exists }(x, A) \in U \text { with } \\
\rho((x, A),(a, A))<2 \rho((x, A), A \times\{A\})\} .
\end{array}
$$

To show that $W_{U}$ is open in $K$ we note that the functions $(a, A) \mapsto(x, A)$ for fixed $x$ and $(a, A) \mapsto A \times\{A\}$ are continuous. Then the set

$$
W_{x}=\{(a, A) \in K \mid \rho((x, A),(a, A))<2 \rho((x, A), A \times\{A\})\}
$$

is open, and so is $W_{U}=\bigcup_{x \in U} W_{x}$.

Note that if $\left\{U_{i}\right\}$ is a sequence in $\mathcal{U}$ and $(a, A) \in K \cap \liminf U_{i}$, then

$$
\lim _{i} W_{U_{i}}=\{(a, A)\} \text {. }
$$

Let $\mathcal{V}$ be a clopen, pairwise disjoint cover of $Y \backslash m^{-1}(K)$ such that $\mathcal{V}$ refines $m^{-1}(\mathcal{U})$. For $V \in \mathcal{V}$ let $U_{V} \in \mathcal{U}$ with $V \subset m^{-1}\left(U_{V}\right)$. Define a set-valued function $F: Y \rightarrow X \times \exp _{b}(X)$ as follows. Let $z \in Y$. If $z \notin m^{-1}(K)$ there exists a unique $V \in \mathcal{V}$ with $z \in V$. Let

$$
F(z)=\left\{\begin{array}{l}
\{m(z)\} \text { if } m(z) \in K \\
\left\{(a, A) \in W_{U_{V}}\right\} \text { if } \pi_{2}(m(z))=A \text { and } z \in V \in \mathcal{V}
\end{array}\right.
$$

Lemma 3.2. The map $F$ is lower semicontinuous.

Proof. Let $\left\{z_{i}\right\}$ converge to $z$ in $Y$. Suppose first that $z \notin m^{-1}(K)$. Let $V$ be the unique element of $\mathcal{V}$ such that $z \in V$. Since $V$ is open we may suppose that $z_{i} \in V$ for each $i$. Let $m(z)=(x, A)$ and $m\left(z_{i}\right)=\left(x_{i}, A_{i}\right)$. Since $m$ is continuous, $\lim m\left(z_{i}\right)=m(z)$. If $(a, A) \in F(z)$, then $(a, A) \in W_{U_{V}}$ and $a \in A$. Since $\lim A_{i}=$ $A$, there exists $a_{i} \in A_{i}$ such that $\lim a_{i}=a$. For large $i,\left(a_{i}, A_{i}\right) \in W_{U_{V}}$ since $W_{U_{V}}$ is open. So $\left(a_{i}, A_{i}\right) \in F\left(z_{i}\right)$ for large $i$ and $\lim \left(a_{i}, A_{i}\right)=(a, A)$ as required.

Now assume that $z \in m^{-1}(K)$ and let $m(z)=(x, A)$ where $x \in A$. Then $F(z)=(x, A)$. By $(1)(x, A) \in \lim F\left(z_{i}\right)$. So $F$ is lower semicontinuous. 
Define a set-valued function $\bar{F}: Y \rightarrow X \times \exp _{b}(X)$ by setting $\bar{F}(z)=\overline{F(z)}$ for $z \in Y$. Then, $\bar{F}$ is also lower semicontinuous and has closed values in the complete metric space $\left(X \times \exp _{b}(X), \rho\right)$ (see [5, page 298, 4.5.23(c)]). By the 0-dimensional Michael selection theorem [8] $\bar{F}$ has a continuous selection $\varphi: Y \rightarrow X \times \exp _{b}(X)$. Note that $\varphi\left(m^{-1}(x, A)\right)=\{(x, A)\}$ if $(x, A) \in K$.

Define an operator $e: C_{b}^{*} \rightarrow \mathbb{R}^{X}$ by setting, for $g \in C_{b}^{*}$ and $x \in X$,

$$
e(g)(x)=\int_{m^{-1}(x, \operatorname{dom} g)} g\left(\pi_{1}(\varphi(z))\right) d \mu_{(x, \operatorname{dom} g)} .
$$

The rest of the proof of Theorem 3.1 will consist of verification of the properties of the map $e$, which is done in the following lemmas.

Lemma 3.3. For every $g \in C_{b}^{*}$ the function $e(g)$ is bounded and continuous on $X$.

Proof. The fact that $e(g)$ is a continuous, bounded function on $X$ follows from continuity and boundedness of the function $g \circ \pi_{1} \circ \varphi$ on $m^{-1}(X \times\{\operatorname{dom} g\})$ and continuity of the measures. Indeed, for a sequence $\left\{x_{i}\right\}$ from $X$ converging to some $x_{0} \in X$, let $\left(x_{i}, \operatorname{dom} g\right)=\alpha_{i},\left(x_{0}, \operatorname{dom} g\right)=\alpha$ and $X \times\{\operatorname{dom} g\}=T$. We obtain

$$
\begin{aligned}
e(g)\left(x_{i}\right)= & \int_{m^{-1}\left(\alpha_{i}\right)} g\left(\pi_{1}(\varphi(z))\right) d \mu_{\alpha_{i}}=\int_{m^{-1}(T)} g\left(\pi_{1}(\varphi(z))\right) d \mu_{\alpha_{i}} \overrightarrow{i \rightarrow \infty} \\
& \int_{m^{-1}(T)} g\left(\pi_{1}(\varphi(z))\right) d \mu_{\alpha}=\int_{m^{-1}(\alpha)} g\left(\pi_{1}(\varphi(z))\right) d \mu_{\alpha}=e(g)\left(x_{0}\right) .
\end{aligned}
$$

Lemma 3.4. The map e is a linear, regular extension operator.

Proof. If $x \in \operatorname{dom} g$, then $\varphi\left(m^{-1}(x, \operatorname{dom} g)\right)=\{(x, \operatorname{dom} g)\}$ and so

$$
e(g)(x)=\int_{m^{-1}(x, \operatorname{dom} g)} g(x) d \mu_{(x, \operatorname{dom} g)}=g(x) .
$$

Therefore, $e$ is an extension operator. Since integration is a linear operation, $e$ is linear.

From the definition of $e$ we see that $\|e(g)\| \leq\|g\|$ for every $g \in C_{b}^{*}$ because

$$
\mu_{(x, \operatorname{dom} g)}\left(m^{-1}(x, \operatorname{dom} g)\right)=\mu_{(x, \operatorname{dom} g)}(Y)=1
$$

for every $x \in X$. And since $e(g)$ is an extension of $g$ we get $\|e(g)\|=\|g\|$. Also, it is clear that $e$ maps $\mathbf{1}_{A}$ to $\mathbf{1}_{X}$ for every $A \in \exp _{b}(X)$. Therefore, $e$ is regular.

Lemma 3.5. The map e is continuous with respect to the topology of uniform convergence on compact sets on $C^{*}(X)$.

Proof. In fact, it is enough to show that if a sequence $\left\{g_{n}\right\}$ converges to $g$ in $C_{b}^{*}$ and if $\left\{x_{n}\right\}$ is a sequence in $X$ converging to $x \in X$, then the sequence $\left\{e\left(g_{n}\right)\left(x_{n}\right)\right\}$ converges to $e(g)(x)$. This is the condition of continuous convergence of functions, which for metric spaces is equivalent to uniform convergence on compact sets (see [10, page 109]). Suppose that $\left\{g_{n}\right\}$ converges to $g$ in $C_{b}^{*}$ and $\left\{x_{n}\right\}$ converges to $x$ in $X$, and write $\left(x_{n}, \operatorname{dom} g_{n}\right)=\beta_{n}$ and $(x, \operatorname{dom} g)=\beta$. 
Case 1. Suppose $\beta_{n}=\beta$ for every $n \in \mathbb{N}$. Since all the functions $g_{n}$ and $g$ have the same domain and their graphs converge in the Hausdorff metric, we conclude that $\left\{g_{n}\right\}$ converges pointwise to $g$ on dom $g$ (see [9]). Then the sequence $\left\{g_{n} \circ \pi_{1} \circ \varphi\right\}$ and the limit function $g \circ \pi_{1} \circ \varphi$ satisfy the hypothesis of Lebesgue's dominated convergence theorem and we obtain $e\left(g_{n}\right)\left(x_{n}\right)=e\left(g_{n}\right)(x) \rightarrow e(g)(x)$.

Case 2 . Now suppose that $\beta_{n} \neq \beta$ for every $n \in \mathbb{N}$. Then by passing to a subsequence if necessary, we may suppose $\beta_{n} \neq \beta_{k}$ for $n \neq k$. Let

$$
Y^{\prime}=m^{-1}(\beta) \cup \bigcup_{n=1}^{\infty} m^{-1}\left(\beta_{n}\right) .
$$

Define a function $h: Y^{\prime} \rightarrow \mathbb{R}$ as follows:

$$
h(z)=\left\{\begin{array}{l}
g\left(\pi_{1}(\varphi(z))\right) \text { if } z \in m^{-1}(\beta) \\
g_{n}\left(\pi_{1}(\varphi(z))\right) \text { if } z \in m^{-1}\left(\beta_{n}\right) .
\end{array}\right.
$$

Since the sequence $\left\{\Gamma_{g_{n}}\right\}$ converges to $\Gamma_{g}$ in the Hausdorff metric, one can show that if a sequence $\left\{a_{n}\right\}$ in $X$ converges to $a \in \operatorname{dom} g$ and $a_{n} \in \operatorname{dom} g_{n}$ for each $n$, then $g_{n}\left(a_{n}\right)$ converges to $g(a)$. Using this condition we conclude that $h$ is a continuous map on $Y^{\prime}$. Since the measures $\left\{\mu_{\beta_{n}}\right\}_{n=1}^{\infty}$ converge to $\mu_{\beta}$ we obtain

$$
\begin{aligned}
e\left(g_{n}\right)\left(x_{n}\right)= & \int_{m^{-1}\left(\beta_{n}\right)} g_{n}\left(\pi_{1}(\varphi(z))\right) d \mu_{\beta_{n}}=\int_{Y^{\prime}} h(z) d \mu_{\beta_{n}} \overrightarrow{n \rightarrow \infty} \\
& \int_{Y^{\prime}} h(z) d \mu_{\beta}=\int_{m^{-1}(\beta)} g\left(\pi_{1}(\varphi(z))\right) d \mu_{\beta}=e(g)(x) .
\end{aligned}
$$

To prove the convergence for any sequence $\left\{\left(x_{n}, g_{n}\right)\right\}$ we will have to pass to a subsequence to which Case 1 or Case 2 applies. Therefore, $e\left(g_{n}\right)\left(x_{n}\right) \rightarrow e(g)(x)$.

Combining the results from Lemma 3.3. Lemma 3.4 and Lemma 3.5 we see that $e$ satisfies all the conditions claimed.

\section{Remarks and open questions}

Remark 4.1. If $A \in \exp _{b}(X)$ and a sequence of partial functions $\left\{g_{n}\right\}$ in $C^{*}(A)$ converges uniformly to $g \in C^{*}(A)$ on $A$, then the sequence of extensions $e\left(g_{n}\right)$ converges uniformly to $e(g)$ on $X$. Indeed, since $e$ is linear and preserves norms, we obtain

$$
\left\|e(g)-e\left(g_{n}\right)\right\|=\left\|e\left(g-g_{n}\right)\right\|=\left\|g-g_{n}\right\| \underset{n \rightarrow \infty}{\longrightarrow} 0 .
$$

Remark 4.2. It is known that in general there is no analogue of the Dugundji theorem on linear extensions of continuous, real functions defined on a closed subset of a metric space for the case of uniformly continuous functions (see [11, Remarks in $\S 2]$ ). Since Hausdorff metric convergence of graphs of uniformly continuous functions with common domain is equivalent to uniform convergence of these functions, we conclude that in general there is no linear extension operator from $C_{u b}^{*}$ to $C_{u}^{*}(X)$ which is continuous with respect to the topology of uniform convergence on $C_{u}^{*}(X)$.

The following question arises naturally:

Question 4.3. Suppose that $(X, d)$ is bounded. Does there exist a linear, regular extension operator $u$ from $C_{b}^{*}$ to $C^{*}(X)$ such that $\Gamma_{u\left(g_{n}\right)}$ converges to $\Gamma_{u(g)}$ in the Hausdorff metric whenever $\left\{g_{n}\right\}$ converges to $g$ in $C_{b}^{*}$ ? 


\section{ACKNOWLedGement}

The authors wish to express gratitude to the referee for useful suggestions and remarks which helped to improve the paper.

\section{REFERENCES}

[1] S. Ageev and E. D. Tymchatyn, On exact Milyutin maps, Topology Appl. 153 (2005), 227238. MR2175347 (2006h:54008)

[2] G. Beer, Metric spaces on which continuous functions are uniformly continuous and Hausdorff distance, Proc. Amer. Math. Soc. 95 (1985), 653-658. MR810180 (87e:54024)

[3] T. Banakh, N. Brodskiy. I. Stasyuk and E. D. Tymchatyn, On continuous extension of uniformly continuous functions and metrics, Colloq. Math. 116 (2009), 191-202. MR2520139

[4] J. Dugundji, An extension of Tietze's theorem, Pacific J. Math. 1 (1951), 353-367. MR0044116 (13:373c)

[5] R. Engelking, General Topology, Heldermann, Berlin, 1989. MR1039321(91c:54001)

[6] H. P. Kunzi and L. B. Shapiro, On simultaneous extension of continuous partial functions, Proc. Amer. Math. Soc. 125 (1997), 1853-1859. MR1415348(98g:54015)

[7] K. Kuratowski, Sur l'espace des fonctions partielles, Ann. Mat. Pura Appl. 40 (1955), 61-67. MR0074807 (17:650b)

[8] E. Michael, Selected selection theorems, Amer. Math. Monthly 63 (1956), 233-238. MR.1529282

[9] S. A. Naimpally, Graph topology for function spaces, Trans. Amer. Math. Soc. 123 (1966), 267-272. MR0192466 (33:691)

[10] L. Narici and E. Beckenstein, Topological vector spaces, Pure and Applied Mathematics 95, Marcel Dekker Inc., New York-Basel, 1985. MR812056 (87c:46003)

[11] A. Pelczynski, Linear extensions, linear averagings, and their applications to linear topological classification of spaces of continuous functions, Dissertationes Math. (Rozprawy Mat.) 58 (1968). MR0227751(37:3335)

[12] E. N. Stepanova, Continuation of continuous functions and the metrizability of paracompact p-spaces, Mat. Zametki 53 (1993), 92-201. Translation in Math. Notes 53 (1993), 308-314. MR94k:54031

[13] E. D. Tymchatyn and M. Zarichnyi, On simultaneous linear extensions of partial (pseudo) metrics, Proc. Amer. Math. Soc. 132 (2004), 2799-2807. MR2054807 (2005d:54032)

Faculty of Science, Shizuoka University, 836 Ohya 422-8059, Shizuoka, Japan

E-mail address: sakoyam@ipc.shizuoka.ac.jp

Department of Mechanics and Mathematics, Lviv National University, UniversytetSka St. 1, Lviv 79000, Ukraine

Current address: Department of Computer Science and Mathematics, Nipissing University, 100 College Drive, Box 5002, North Bay, ON, P1B 8L7, Canada

E-mail address: i_stasyuk@yahoo.com

Department of Mathematics and Statistics, University of Saskatchewan, McLean Hall, 106 Wiggins Road, Saskatoon, SK S7N 5E6, Canada

E-mail address: tymchat@math.usask.ca

Institute for Applied Problems of Mechanics and Mathematics, Ukrainian Academy of Sciences, 3в Naukova St., Lviv 79060, Ukraine

Current address: Prycarpathian National University, Ivano-Frankivsk, Ukraine

E-mail address: andriyzag@yahoo.com 